\title{
Secondary bifurcations of hexagonal patterns in a nonlinear optical system: Alkali metal vapor in a single-mirror arrangement
}

\author{
Damià Gomila, ${ }^{1}$ Thorsten Ackemann, ${ }^{2}$ Edgar Grosse Westhoff, ${ }^{2}$ Pere Colet, ${ }^{1}$ and Wulfhard Lange ${ }^{2}$ \\ ${ }^{1}$ Institut Mediterrani d'Estudis Avançats, IMEDEA* (CSIC-UIB), Campus Universitat Illes Balears, \\ E-07071 Palma de Mallorca, Spain \\ ${ }^{2}$ Institut für Angewandte Physik, Westfälische Wilhelms-Universität Münster, Corrensstrasse 2/4, D-48149 Münster, Germany
}

(Received 26 February 2003; published 24 March 2004)

\begin{abstract}
Secondary bifurcations of hexagonal patterns are analyzed in a model of a single-mirror arrangement with an alkali metal vapor as the nonlinear medium. A stability analysis of the hexagonal structures is performed numerically. Different instabilities are predicted in dependency on the wave number of the hexagons. Some of the instabilities take place at a finite wave number and result in the formation of structures with 12 spatial modes. These structures are compared with those observed experimentally.
\end{abstract}

DOI: 10.1103/PhysRevE.69.036205 PACS number(s): 89.75.Kd, 42.65.Sf, 47.54.+r, 47.20.Ky

\section{INTRODUCTION}

Stripes, rhombi, squares, and hexagons are typical periodic patterns widely studied in two-dimensional dissipative systems [1]. Periodic patterns with more than six spatial modes have been the subject of considerable theoretical interest [2-6]. They have been observed experimentally in parametrically excited hydrodynamical systems (Faraday instability [7-11]) and in nonlinear optical systems, first with an externally imposed discrete rotational symmetry [12], and, more recently, in a system with continuous rotational symmetry $[13,14]$.

In the latter system, these structures appear via a secondary bifurcation from a primary hexagonal structure in a broad range of parameters $[13,14]$. Generic secondary bifurcations of spatial periodic structures have been classified rather completely in one spatial dimension $[15,16]$. In two-dimensional systems they have been analyzed mainly with a symmetrybased approach $[2,3,5,17]$. Here, we address the problem of secondary bifurcations of hexagonal patterns performing numerically a stability analysis of hexagonal structures in a model of a nonlinear optical system showing such bifurcations $[13,14]$.

Our analysis predicts several possible instabilities of the hexagonal patterns versus different subharmonic wave numbers. The linear and nonlinear stages of the evolution of the instabilities are investigated. The resulting patterns can be either hexagons with a different wave number and/or a different orientation or superlattice patterns consisting of 12 wave vectors. The superlattice patterns are compared with the ones found in the experiment.

The paper is organized as follows. In Sec. II the experimental scheme and the microscopic model are introduced. In Sec. III a linear stability analysis of the homogeneous solution is performed. In Sec. IV we find the stationary hexagonal pattern solutions of the model. Then, in Sec. V, we perform a linear stability analysis of the hexagonal patterns and discuss their different instabilities. In Sec. VI the theoretical

*http://www.imedea.uib.es/PhysDept. results are compared with the experimental findings. Finally, in Sec. VII we give some concluding remarks.

\section{EXPERIMENTAL SETUP AND MODEL}

The system under study (see Fig. 1) is based on a single feedback mirror arrangement, which is an archetypal system for optical pattern formation [18-20]. A thin nonlinear optical medium is irradiated by a laser beam which is homogeneous in amplitude and phase. The transmitted beam is retroreflected into the medium by a plane high-reflectivity mirror placed at a distance $d$ behind the medium. During the propagation of the light field to the mirror and back, different points in the transverse plane are coupled by diffraction.

A spatial instability might arise, because a fluctuation of the refractive index inside the medium causes a phase modulation of the transmitted wave. Due to diffraction, this phase modulation is — at least partially_converted to an amplitude modulation. Under suitable conditions this amplitude modulation can reinforce the original fluctuation of the refractive index, which is assumed to be intensity dependent. Thus a macroscopic modulation - a pattern-can develop in the transverse cross section of the laser beam as well as in the transverse distribution of the refractive index within the medium [18-20].

In the system we are going to discuss, sodium vapor in a nitrogen buffer gas atmosphere is used as the nonlinear medium. The input light field is circularly polarized and its frequency is tuned to the vicinity of the $D_{1}$ line. The nonlinearity stems from optical pumping between Zeeman sublevels of the ground state $[21,22,20]$. If the system is suitably prepared, it is possible to describe the light-matter interaction

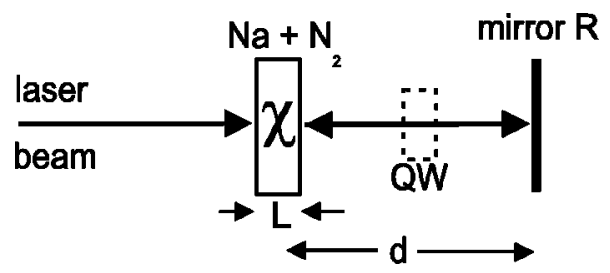

FIG. 1. Scheme of the investigated nonlinear optical system. See text. 
in the framework of a simple two-level model with degeneracy $\left(J=1 / 2 \rightarrow J^{\prime}=1 / 2\right.$-transition) $[22,20,23]$. Then the decisive dynamical variable is the orientation $w$ which is the normalized population difference between the two Zeeman sublevels of the ground state $[22,20,23]$. The $z$ axis is chosen to be parallel to the direction of the wave vector of the input light field. $x, y$ denote the coordinates transverse to the direction of propagation. Then a longitudinally averaged orientation $\phi$ is introduced by defining (see also Ref. [24])

$$
\phi(x, y, t)=\frac{1}{L} \int_{0}^{L} w(x, y, z, t) d z .
$$

Here $L$ denotes the length of the medium in $z$-direction. In the following, we will discuss a modified system in which there is an additional polarization changing element-a quarter-wave plate-in the feedback loop. This system has a rather low threshold $[23,25]$ and displays interesting secondary bifurcations of hexagons $[26,13,14]$.

The dynamics of $\phi$ is governed by the partial differential equation $[22,20,23]$

$$
\begin{aligned}
\partial_{t} \phi= & -\left(\gamma-D \nabla^{2}\right) \phi+\frac{1}{2 L \alpha_{0}} \\
& \times\left[\left(1-e^{-2 L \alpha_{0}(1-\phi)}\right) P^{0}-\left(1-e^{-2 L \alpha_{0}(1+\phi)}\right) P^{0} R\right. \\
& \left.\times\left|e^{-i\left(d / k_{0}\right) \nabla^{2}} e^{-L \alpha_{0}(1-i \bar{\Delta})(1-\phi)}\right|^{2}\right] .
\end{aligned}
$$

$\gamma$ models relaxation by collisions and $D \nabla^{2}$ gives the thermal motion of the atoms ( $\nabla^{2}$ is the Laplacian in transverse space and $D$ the diffusion constant). The parameter $\alpha_{0}$ parametrizes the optical depth of the medium, it is half the smallsignal absorption coefficient. $\bar{\Delta}$ denotes the detuning of the incident laser beam with respect to the $D_{1}$ resonance. It is normalized to the linewidth (half width at half maximum) and is positive, if the laser frequency is higher than the resonance frequency. $k_{0}=2 \pi / 589.6 \mathrm{~nm}$ is the wave number of the input light field in vacuum and $R$ the reflectivity of the mirror. The pump rate $P^{0}$ is proportional to the intensity of the input light field. It is also the main bifurcation parameter. The two exponentials in the last term of Eq. (2) describe diffraction and the action of the medium on phase and amplitude of the light field. Details on the system and on the derivation of Eq. (2) can be found in Refs. [22,20,23,25].

In this paper, only the case of a plane wave input field is discussed. We remark that for a description of a spatially varying input field Eq. (2) needs to be modified slightly.

\section{LINEAR STABILITY ANALYSIS OF THE HOMOGENEOUS SOLUTION}

The steady state plane wave solution $\phi_{0}$ of Eq. (2) is implicitly given by

$$
\begin{aligned}
2 \gamma L \alpha_{0} \phi_{0}= & P^{0}\left[\left(1-e^{-2 L \alpha_{0}\left(1-\phi_{0}\right)}\right)\right. \\
& \left.-R\left(1-e^{-2 L \alpha_{0}\left(1+\phi_{0}\right)}\right) e^{-2 L \alpha_{0}\left(1-\phi_{0}\right)}\right] .
\end{aligned}
$$

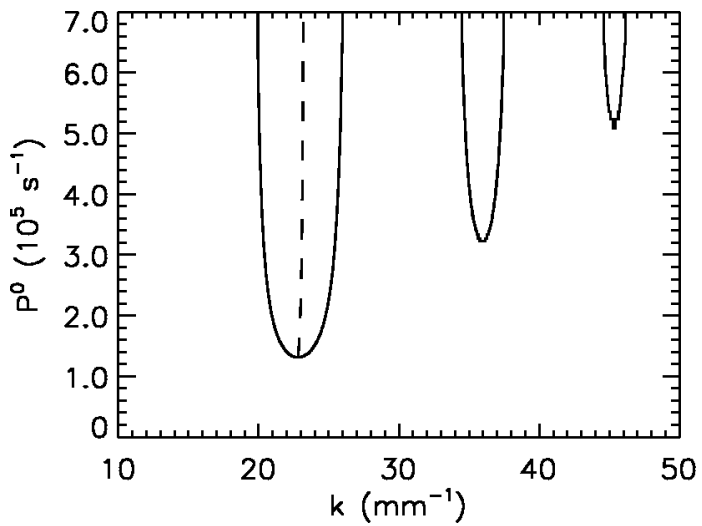

FIG. 2. The solid lines indicate the values of the pump $P^{0}$ for which a periodic perturbation of wave number $k$ becomes unstable. The dashed line indicates the wave number with maximum growth rate. Here $\gamma=1.5 \mathrm{~s}^{-1}, D=0.0003563 \mathrm{~m}^{2} \mathrm{~s}^{-1}, L \alpha_{0}=2.595, R$ $=0.915, d / k_{0}=8.258 \times 10^{9} \mathrm{~m}^{2}$, and $\bar{\Delta}=4.0$. For these values of the parameters the instability threshold is at $P^{0} \simeq 1.31 \times 10^{5} \mathrm{~s}^{-1}$ and the critical wave number is $k_{c} \simeq 22.8 \mathrm{~mm}^{-1}$.

A linear stability analysis of the homogeneous solution with respect to spatially periodic perturbations yields the dispersion relation

$$
\begin{aligned}
\lambda(k)= & -\gamma-D k^{2}-e^{-2 L \alpha_{0}\left(1-\phi_{0}\right)} P^{0} \\
& -P^{0} R\left\{\left(1-e^{-2 L \alpha_{0}\left(1+\phi_{0}\right)}\right) e^{-2 L \alpha_{0}\left(1-\phi_{0}\right)}\right. \\
& \left.\times\left[\cos \left(\frac{d}{k_{0}} k^{2}\right)+\bar{\Delta} \sin \left(\frac{d}{k_{0}} k^{2}\right)\right]-e^{-4 L \alpha_{0}}\right\},
\end{aligned}
$$

where $\lambda(k)$ is the growth rate of a periodic perturbation of wave number $k$. In Fig. 2 we display the marginal stability curve $[\lambda(k)=0]$.

The homogeneous solution never becomes unstable against homogeneous perturbations $(k=0)$. In fact, for large values of the pump the eigenvalue $\lambda(k=0) \propto-P^{0}$. An important point to notice is that the marginal stability curve has a vertical asymptote for $k_{a} \simeq 1.9 \times 10^{4} \mathrm{~m}^{-1}$ [27], and therefore perturbations of the homogeneous solution with a wave number smaller than $k_{a}$ are never unstable. For pump intensities above but close to threshold the system develops stationary hexagonal patterns [25].

\section{STATIONARY HEXAGONAL PATTERNS}

Stationary hexagonal solutions of the form

$$
\phi_{h}(\vec{x})=\sum_{n=0}^{N-1} \phi_{n} e^{i \vec{k}_{n}^{0} \cdot \vec{x}}
$$

where $\vec{k}_{n}^{0}$ are the wave vectors of the pattern including the homogeneous component $\vec{k}_{0}^{0}, \phi_{n}$ are complex coefficients, and $N$ is the number of Fourier modes, can be found by solving the stationary form $\left(\partial_{t} \phi_{h}=0\right)$ of Eq. (2). A set of coupled nonlinear equations for the amplitudes of the Fourier 

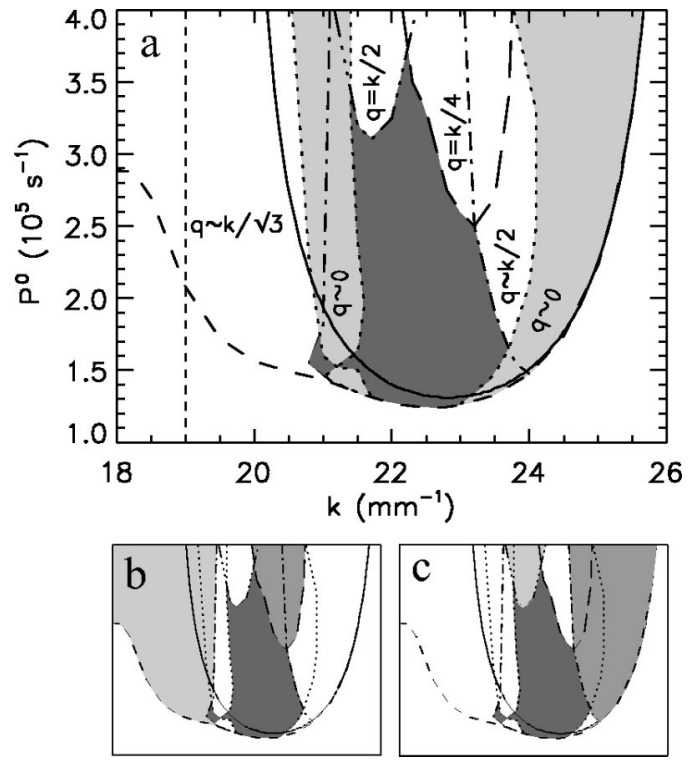

FIG. 3. Marginal stability diagram of the hexagonal patterns. Stationary hexagons with a wave number $k$ exist above the lowest dashed line. The solid line is the first instability region shown in Fig. 2 and the vertical dashed line on the left is the vertical asymptote $k_{a}$. The dark gray area indicates the region in the $P^{0}-k$ diagram where the stationary hexagonal patterns are stable (Busse balloon). The other discontinuous lines indicate the threshold for different instabilities of the hexagonal solutions. The light gray areas in panel (a) indicate the regions where hexagonal patterns are unstable against long-wavelength perturbations (Sec. V A). In the panel (b), the dark gray area is the stable region as in panel (a) while the gray and light gray areas correspond to the $q=k / 4$ (Sec. V D) and $q$ $\sim k / \sqrt{3}$ (Sec. V B) instability regions. In (c), the dark gray area is also the stable region, and the instability regions $q \sim k / 2$ (Sec. V E) and $q=k / 2$ (Sec. V C) are indicated in gray and light gray.

components $\phi_{n}$ is obtained. Since $\phi_{h}$ is real, $\phi_{n}=\phi_{m}^{*}$ if $\vec{k}_{n}^{0}$ $=-\vec{k}_{m}^{0}$. We consider the homogeneous component, the six fundamental wave vectors, and 84 harmonic modes in Fourier space. This amounts to a total of $N=91$ modes and covers a range of wave numbers up to five times the wave number of the fundamental modes. The higher harmonics have to be considered since the bifurcation to hexagons is generally subcritical and, hence, higher harmonics have finite amplitudes even at threshold. Starting from a suitably chosen initial condition, a Newton-Raphson method is used to find solutions of the set of nonlinear equations. With this method we can look for stationary hexagonal solutions with different fundamental wave numbers $k$.

The lowest dashed line in Fig. 3(a) indicates the minimum value of the pump for which stationary hexagonal pattern solutions exist around the first instability region (solid line). It corresponds to a saddle-node bifurcation where an eigenvalue of the hexagonal pattern branch becomes zero as a result of the collision with the unstable branch coming from the homogeneous solution. Typically, a hexagonal branch with a given $k$ starts subcritically from the homogeneous solution at the pump value where $\lambda(k)=0$ (solid line). Such a behavior is consistent with the general theory of hexagon formation: the bifurcation of the homogeneous state to hexa- gons is transcritical but the bifurcating branches are unstable until a saddle-node bifurcation point is encountered on the backwardly bifurcating branch [1,28,29]. Hexagons with a wave number much smaller than the critical one have a very large subcritical region. However, the size of the subcritical region becomes progressively smaller for hexagons with larger wave numbers. For hexagons with a wave number somehow larger than $k_{c}$, the dashed line in Fig. 3(a) approaches asymptotically the marginal stability curve of the homogeneous solution (solid line), indicating that the quadratic terms in the normal form are becoming progressively small for large wave numbers. The region of existence of stationary hexagons also extends to the left of the vertical asymptote $k_{a}$ of the marginal stability curve [Fig. 3(a), see part at low wave numbers]. This implies the existence of stationary hexagons with wave numbers that never become linearly unstable from the homogeneous solution. However, as we will see in the following section, both kinds of hexagons - the ones with very large wave numbers and the ones with wave numbers below the vertical asymptote $(k$ $<k_{a}$ ) —are always unstable.

\section{LINEAR STABILITY ANALYSIS OF STATIONARY HEXAGONAL PATTERNS}

In this section, following Ref. [30], we perform the linear stability analysis of the stationary hexagonal solutions found in the preceding section in order to determine their stability as a function of the input power and the fundamental wave number $k$ of the pattern. Linearizing Eq. (2) around the stationary solution (5) the following equation for the fluctuations $\delta \phi(\vec{x}, t)=\phi(\vec{x}, t)-\phi_{h}(\vec{x})$ is obtained

$$
\begin{aligned}
\partial_{t} \delta \phi= & -\left(\gamma-D \nabla^{2}\right) \delta \phi-P^{0}\left[e^{-2 L \alpha_{0}\left(1-\phi_{h}\right)}\right. \\
& \left.+R \mathrm{e}^{-2 L \alpha_{0}\left(1+\phi_{h}\right)}\left|e^{-i\left(d / k_{0}\right) \nabla^{2}} e^{-L \alpha_{0}(1-i \bar{\Delta})\left(1-\phi_{h}\right)}\right|^{2}\right] \delta \phi \\
& -\frac{P^{0} R}{2}\left[(1+i \bar{\Delta})\left(1-e^{-2 L \alpha_{0}\left(1+\phi_{h}\right)}\right)\right. \\
& \times\left(e^{-i\left(d / k_{0}\right) \nabla^{2}} e^{-L \alpha_{0}(1-i \bar{\Delta})\left(1-\phi_{h}\right)}\right) e^{i\left(d / k_{0}\right) \nabla^{2}} \\
& \left.\times\left(e^{-L \alpha_{0}(1+i \bar{\Delta})\left(1-\phi_{h}\right)} \delta \phi\right)+\text { c.c. }\right],
\end{aligned}
$$

which can be written as $\partial_{t} \delta \phi=L\left(\phi_{h}\right) \delta \phi$, where $L\left(\phi_{h}\right)$ is the Jacobian matrix. Since $L$ is a linear operator with periodic coefficients, a general bounded solution can be found which has a Floquet form [15]:

$$
\delta \phi(\vec{x}, t)=\int_{-\infty}^{+\infty} e^{i \vec{q} \cdot \vec{x}} \delta \widetilde{\phi}(\vec{x}, \vec{q}, t) d \vec{q},
$$

where $\delta \widetilde{\phi}(\vec{x}, \vec{q}, t)$ is a function with the same spatial periodicity as the stationary pattern $\phi_{h}$. Hence, it can be written as

$$
\delta \widetilde{\phi}(\vec{x}, \vec{q}, t)=\sum_{n=0}^{N-1} \delta \phi_{n}(\vec{q}, t) e^{i \vec{k}_{n}^{0} \cdot \vec{x}} .
$$

Since $\delta \phi$ is real, $\delta \widetilde{\phi}(\vec{x}, \vec{q}, t)=\delta \widetilde{\phi}^{*}(\vec{x},-\vec{q}, t)$. From Eqs. (6) and (8) we obtain a set of coupled linear equations for the 
coefficients $\delta \phi_{n}(\vec{q}, t)$. The important point is that perturbations with different vectors $\vec{q}$ are uncoupled. To know the stability of the solution against any possible perturbation it is sufficient to consider only vectors $\vec{q}$ inside the first Brillouin zone of the hexagonal lattice defined by the wave vectors $\vec{k}_{n}^{0}$ of the pattern. Any perturbation with a vector $\vec{q}^{\prime}$ outside the first Brillouin zone is equivalent to another with a vector $\vec{q}$ $=\vec{q}^{\prime}+\vec{k}_{n}^{0}$ inside, where $\vec{k}_{n}^{0}$ is one of the wave vectors of the hexagonal solution. In this way one finds a set of $N$ eigenvalues and eigenvectors for each vector $\vec{q}$. We order the eigenvalues $\lambda_{i}(\vec{q})$ according to the value of their real part $\operatorname{Re}\left[\lambda_{i}(\vec{q})\right]>\operatorname{Re}\left[\lambda_{i+1}(\vec{q})\right]$. When a hexagonal pattern becomes unstable against perturbations with a vector $\vec{q}$, wave vectors at that precise distance from the wave vectors of the pattern, corresponding to the Fourier components of the unstable eigenmode, are expected to grow linearly.

The result of the linear stability analysis of hexagonal patterns with different modulus $k$ of the fundamental wave vectors is shown in Fig. 3. The dark shadowed area indicates the region of stability of the hexagonal solutions (Busse balloon). The different unstable regions are labeled with the modulus $q$ of the perturbation vector $\vec{q}$ that is unstable. The place where two instability lines cross corresponds to a codimension-two bifurcation point. The three most important instabilities are those corresponding to the three regions labeled as $q=k / 2, q=k / 4$, and $q \sim k / 2$. These are the instabilities that hexagons with wave numbers close to the critical one will encounter by increasing the pump. They are analyzed in the following sections.

\section{A. Long-wavelength instability}

In the regions of Fig. 3 labeled as $q \sim 0$ the hexagonal patterns are unstable against long-wavelength perturbations as illustrated in Fig. 4. The largest real part of the eigenvalues is shown for three different values of the input power: below, close to, and above threshold (see, e.g., Refs. [31-34] for previous work on phase instabilities of hexagons). As a result of the instability, wave vectors very close to those of the hexagonal pattern are expected to grow linearly. In an infinite system and close to threshold, an unstable hexagonal pattern changes its wave number and orientation continuously until it reaches the stable region. In finite size systems, the discretization in Fourier space imposes a lower limit to the change of modulus and orientation of the wave vectors of a hexagonal pattern. The growth of the Fourier modes of the grid closest to those of the unstable hexagonal pattern leads to a discrete change in the wave number and orientation of the pattern towards the stable region. Figure 5 shows the time evolution of an unstable stationary hexagonal pattern with $k=23.5 \mathrm{~mm}^{-1}$ and $P^{0}=1.35 \times 10^{5} \mathrm{~s}^{-1}$ in a rather large system. In the first stages of the time evolution wave vectors very close to those of the pattern grow linearly in time. As soon as these wave vectors get a large amplitude they deform the pattern in the real space with a long-wavelength modulation (second and third snapshots) and, finally, the original pattern is replaced by another one with a slightly smaller wave number (fourth snapshot) inside the stable region $(k$ $=22.2 \mathrm{~mm}^{-1}$ ).
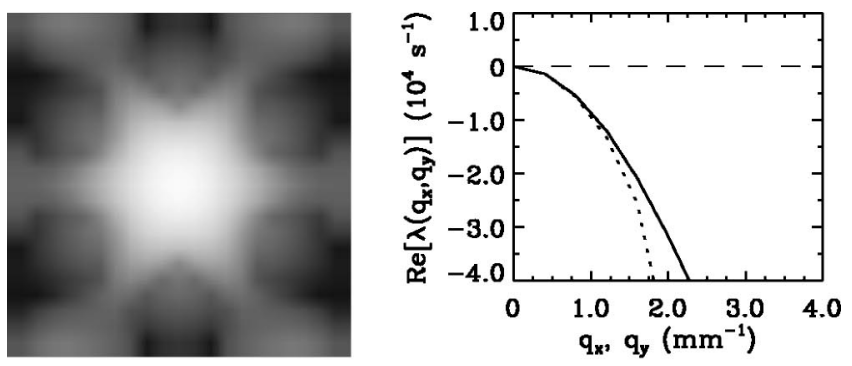

(a)
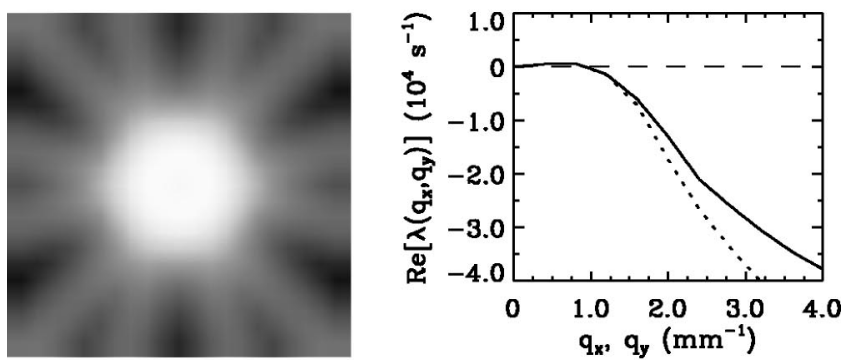

(b)
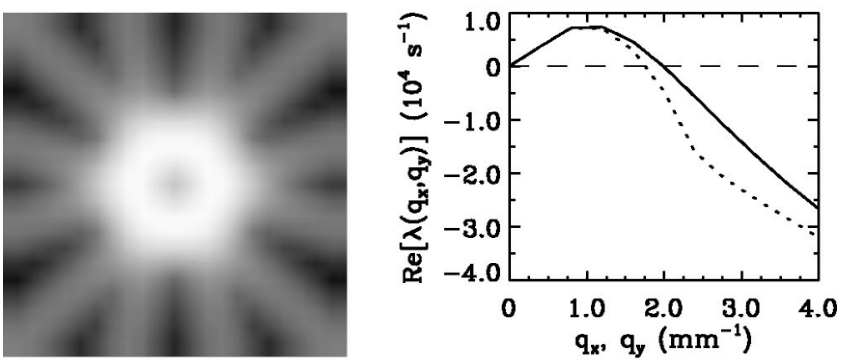

(c)

FIG. 4. Left: largest real part of the eigenvalues $\operatorname{Re}\left[\lambda_{0}\left(q_{x}, q_{y}\right)\right]$ of a stationary hexagonal pattern of fundamental wave number $k$ $=23.5 \mathrm{~mm}^{-1}$. As shown in Fig. 3(a), for this value of $k$ the hexagonal pattern becomes unstable decreasing the pump at $P_{\text {eck }}^{0}$ $=1.47 \times 10^{5} \mathrm{~s}^{-1}$ (from top to bottom $P^{0}=1.95 \times 10^{5} \mathrm{~s}^{-1}, 1.45$ $\times 10^{5} \mathrm{~s}^{-1}$, and $\left.1.35 \times 10^{5} \mathrm{~s}^{-1}\right)$. In each figure the gray scale is taken between the minimum (black) and maximum (white) values of $\operatorname{Re}\left[\lambda_{0}\left(q_{x}, q_{y}\right)\right]$. The center of the figures corresponds to $\vec{q}=0$, where $\lambda_{0}(0,0)=0$. In the first two figures the center represents the maximum value. In the last figure the center has a gray color because it is surrounded by the positive eigenvalues corresponding to the long-wavelength unstable modes. Right: transverse cut of $\operatorname{Re}\left[\lambda_{0}\left(q_{x}, q_{y}\right)\right]$ in the $q_{x}$ (solid line) and $q_{y}$ direction (dotted line).

Far from the threshold of the long-wavelength instability the dispersion relation $\lambda_{0}(\vec{q})$ has a maximum at a finite wave number (Fig. 4, bottom). Therefore, the wave vectors with this maximum growth rate are more likely to dominate the system, and a finite change in the wave number and orientation of an unstable hexagonal pattern may occur even in an infinite system.

The narrow long-wavelength instability region on the left of Fig. 3 can only be observed by considering large systems since in that region only perturbations with a fairly long wavelength have positive growth rate. For sufficiently small systems $(L<6 \mathrm{~mm})$ this instability region is not present and hexagons with small wave numbers are stable until $k$ enters the region labeled as $q \sim k / \sqrt{3}$. 


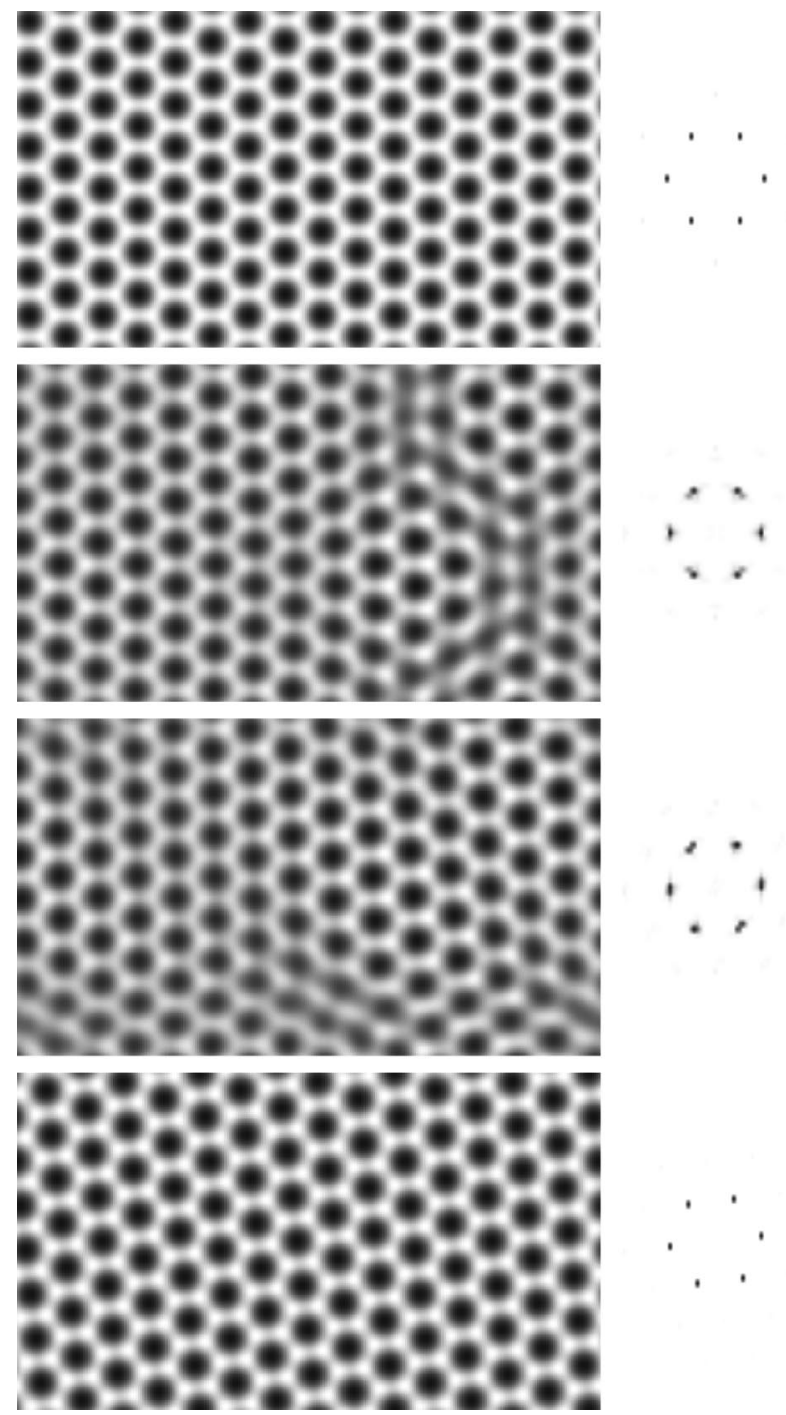

FIG. 5. Time evolution of the orientation $\phi(\vec{x}, t)$ (left) and its Fourier spectra (right) for hexagons unstable against longwavelength perturbations. In the left panels dark (white) color indicates low (large) values of the orientation. In the right panels, the color table is inverted and the white color denotes the background level whereas the black color denotes high amplitude. The dc component is suppressed. The wave number of the initial unstable hexagons is $k=23.5 \mathrm{~mm}^{-1}$ and $P^{0}=1.35 \times 10^{5} \mathrm{~s}^{-1}$. The time increases from top to bottom. Note the growth of Fourier modes close to the fundamental ones and the final state with a smaller wave number $k^{\prime}=22.2 \mathrm{~mm}^{-1}$. This numerical simulation has been performed on a $256 \times 256$ rectangular grid with step width $\Delta k_{x}=k / 16$ and $\Delta k_{y}$ $=\sqrt{3} \Delta k_{x}$. In the following, numerical simulations have been performed on a $128 \times 128$ rectangular grid with $\Delta k_{x}=k / 8$ and $\Delta k_{y}$ $=\sqrt{3} \Delta k_{x}$.

\section{B. $q \sim k / \sqrt{3}$ instability}

In this region [light gray area in Fig. 3(b)] perturbations with $\vec{q}$ close to the vertices of the first Brillouin zone have a positive linear growth rate as shown in Fig. 6 . These vertices are located at a distance $k / \sqrt{3}$ in the direction forming a $30^{\circ}$ angle with the fundamental wave vectors. Strictly speaking the perturbations with positive linear growth are not those

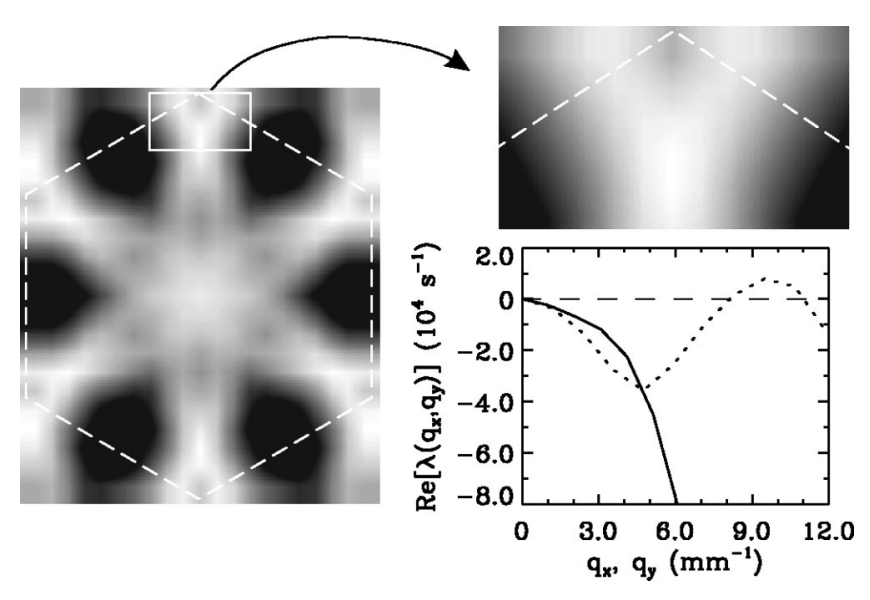

FIG. 6. The same as in Fig. 4 for a hexagonal pattern with $k$ $=20.5 \mathrm{~mm}^{-1}$ in the region $q \sim k / \sqrt{3}$. For this value of $k$, as shown in Fig. 3, the hexagonal pattern is unstable for any value of the pump $P^{0}$. Here we take $P^{0}=1.5 \times 10^{5} \mathrm{~s}^{-1}$. The white dashed hexagon indicates the limits of the first Brillouin zone. Note that the largest eigenvalues are located in the neighborhood of the vertex of the first Brillouin zone. The upper right panel shows an enlargement of the area in the left panel encircled by a solid white line.

with $\vec{q}$ exactly at the vertices of the first Brillouin zone but those located in their surroundings as shown in Fig. 6. If an unstable stationary hexagonal pattern is used as the initial condition in numerical simulations, the growth of wave vectors at a distance of $\sim k / \sqrt{3}$ from the wave vectors of the pattern (forming an angle of about $30^{\circ}$ with these) is observed in the linear stages of the evolution of the instability (Fig. 7, second snapshot). As soon as these modes get a large amplitude, nonlinear dynamics come into play. For low values of the pump, as it is the case in Fig. 7, a hexagonal pattern with the wave number of the linearly unstable modes replaces the original pattern (third and fourth snapshot). The wave number of the new hexagonal pattern lies inside the stable region. At difference with the long-wavelength instability, in this case the wave vectors of the hexagons do not change continuously, but there is a discrete change even in the case of an infinite system. In particular, in addition to the finite change in the modulus, the hexagons rotate almost $30^{\circ}$. For larger values of the pump, the nonlinear dynamics does not lead to the selection of a hexagonal pattern but a more complex nonstationary state with many excited wave vectors. These structures are similar to those obtained for the same values of the pump in calculations starting from random initial conditions.

\section{C. $q=k / 2$ instability}

In the region $q=k / 2$ [light gray area in Fig. 3(c)] the perturbations with vectors $\vec{q}$ of modulus $k / 2$ located at the border of the first Brillouin zone in the direction of the wavevectors of the pattern have a positive growth rate (Fig. 8). Figure 9 shows a simulation where the pump has been increased beyond the instability threshold. In the first stages of the time evolution (second and third snapshots) subharmonic wave vectors with half the wave number of the fundamental modes can be seen growing linearly as predicted by 


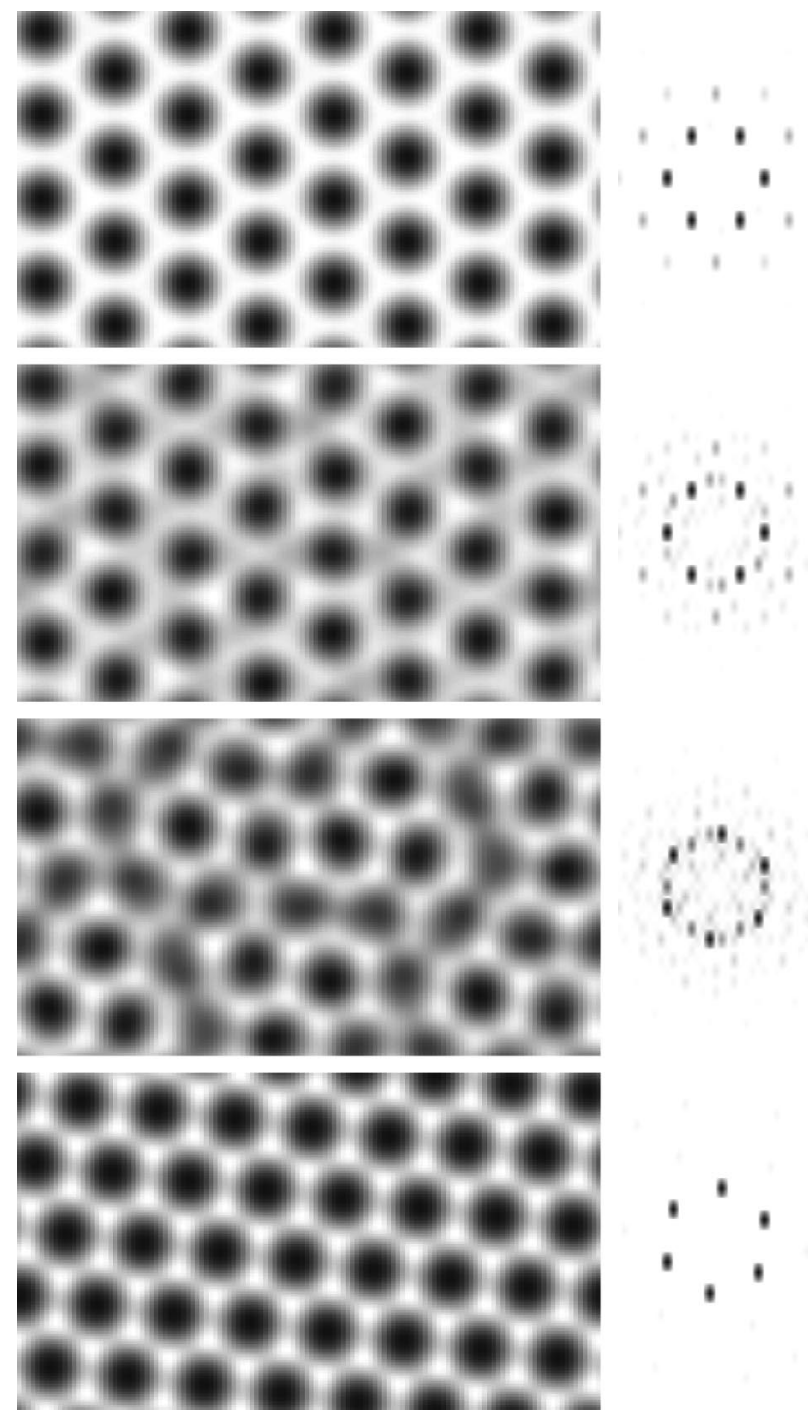

FIG. 7. The same as in Fig. 5 for a hexagonal pattern inside the $q \sim k / \sqrt{3}$ region $\left(k=20.5 \mathrm{~mm}^{-1}, P^{0}=1.5 \times 10^{5} \mathrm{~s}^{-1}\right)$. Note the linear growth of the Fourier modes at a distance $\sim k / \sqrt{3}$ in the $\sim 30^{\circ}$ direction with respect to the fundamental wave vectors of the solution in the second snapshot. The final hexagonal pattern has a wave number $k^{\prime}=22.3 \mathrm{~mm}^{-1}$ which is within the stable region.

the stability analysis. In this case the bifurcation is supercritical since close to the critical point the amplitude of the linearly unstable modes remain small due to nonlinear saturation. As a result of this bifurcation a stationary superlattice is observed. Its periodicity length is two times the one of the underlying primary hexagons (fourth snapshot in Fig. 9).

\section{D. $q=k / 4$ instability}

In the region $q=k / 4$ [gray area in Fig. 3(b)] perturbations with $q=k / 4$ in the direction of the fundamental wave vectors of the pattern have positive growth rate (Fig. 10). These wave vectors can be seen growing in the first stages of the time evolution in a numerical simulation after the pump has been increased above the instability line (Fig. 11). This simulation shows, however, that the transition is subcritical, and the nonlinear dynamics leads to a spatial structure with 12

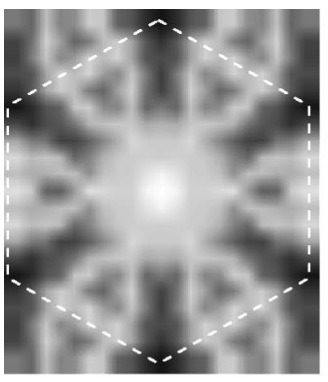

(a)

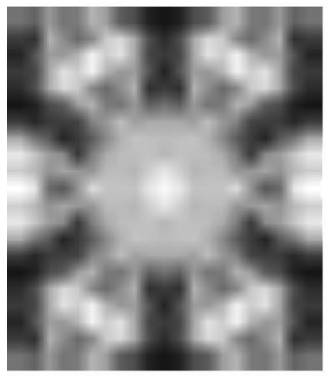

(b)

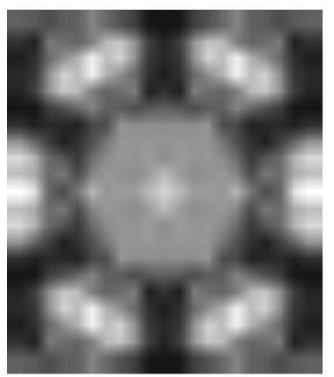

(c)
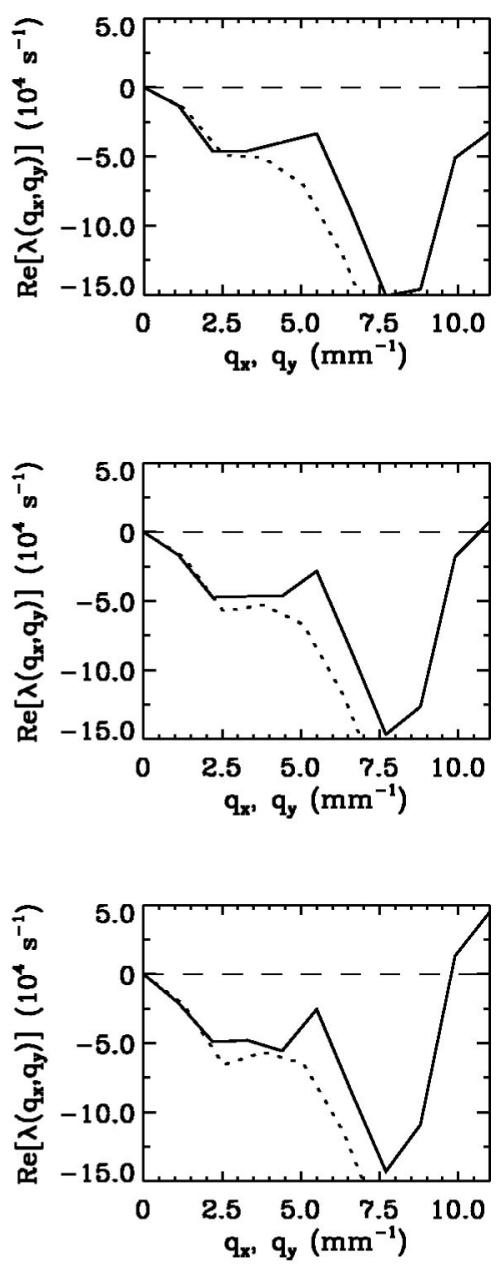

$q_{x}, q_{y}\left(\mathrm{~mm}^{-1}\right)$
FIG. 8. The same as in Fig. 4 for a stationary hexagonal pattern with wave number $k=22.0 \mathrm{~mm}^{-1}$ crossing the instability line of the region $q=k / 2$ located at $P_{k / 2}^{0}=3.25 \times 10^{5} \mathrm{~s}^{-1}$ (from top to bottom $P^{0}=3.0 \times 10^{5} \mathrm{~s}^{-1}, 3.3 \times 10^{5} \mathrm{~s}^{-1}$, and $3.6 \times 10^{5} \mathrm{~s}^{-1}$ ). In the first picture the white dashed line indicates the limits of the first Brillouin zone. Note that the largest eigenvalues are located at the border of the first Brillouin zone in the direction of the fundamental wave vectors of the hexagonal pattern.

fundamental wave vectors. The second hexagonal set has modulus $k=\sqrt{3} k / 2$ and it forms a $30^{\circ}$ angle with the wave vectors of the original pattern (Fig. 11). The resulting structure is a superlattice with a periodicity length twice that of the initial hexagonal pattern. The wave-vector configuration is the same as in Fig. 9 but the relative amplitudes and phases are obviously different.

\section{E. $q \sim k / 2$ instability}

Finally, in the $q \sim k / 2$ region [gray area in Fig. 3(c)], the stationary hexagonal patterns are unstable against perturbations with $\vec{q}$ close to the limits of the first Brillouin zone in the direction of the fundamental wave vectors. However, at difference with the case discussed in Sec. V B, the perturbations that first become unstable are not those with $q=k / 2$ but those in their vicinity (Fig. 12). In the second row of Fig. 12 


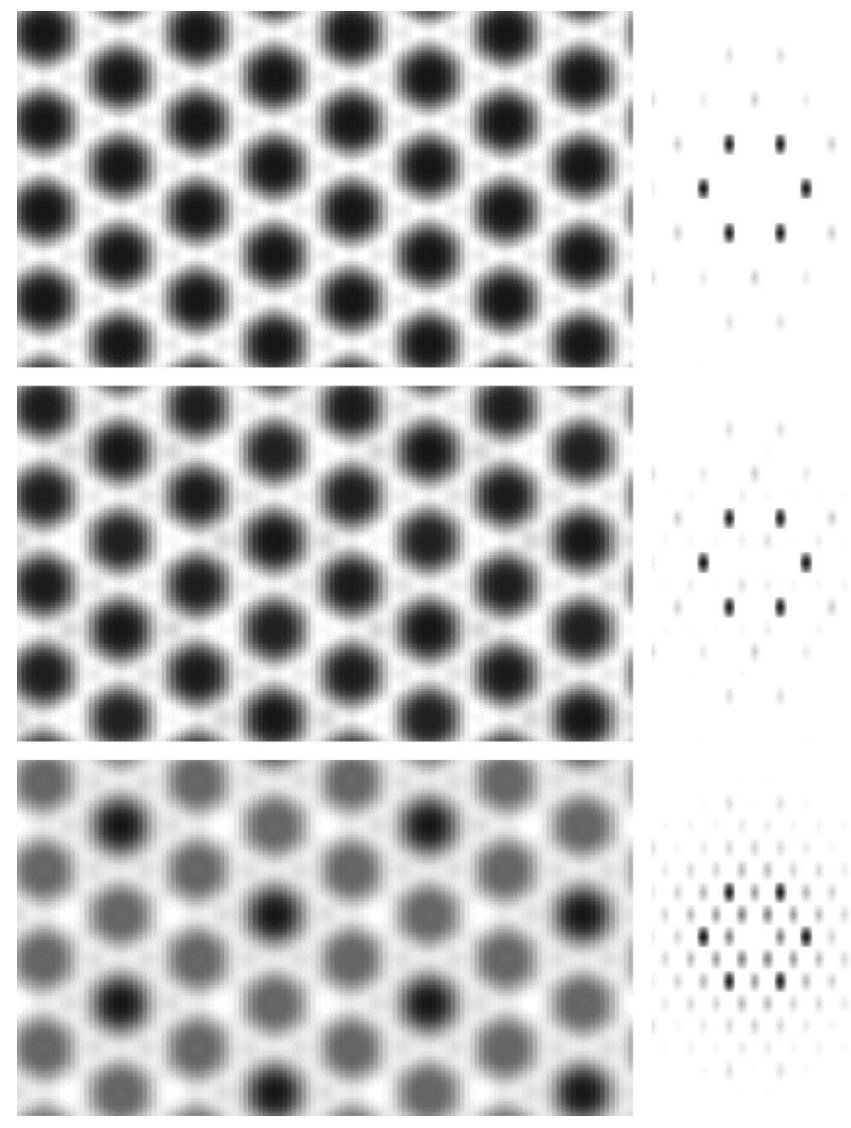

FIG. 9. The same as in Fig. 5 for a hexagonal pattern inside the $q=k / 2$ region $\left(k=22.0 \mathrm{~mm}^{-1}, P^{0}=3.5 \times 10^{5} \mathrm{~s}^{-1}\right)$. Note the linear growth and nonlinear saturation of subharmonics exactly at the half of the wave vectors of the original hexagonal pattern and the final superlattice.

the growth rate of perturbations with $\vec{q}$ close to $\vec{k}_{n}^{0} / 2$ (n $=1,6)$ is already positive while the perturbations with $\vec{q}$ $=\vec{k}_{n}^{0} / 2(n=1,6)$, exactly half of a fundamental wave vector, have negative growth rate as can be seen in the transverse cut. In a numerical simulation Fourier modes at a distance $q \sim k / 2$ in the direction of the fundamental wave vectors are observed to grow, if the pump parameter is increased beyond the instability line (Fig. 13). In contrast to the case $q=k / 2$, the transition is subcritical and drives the system away from the hexagonal pattern solution. In a small system, as it is the case in Fig. 13, the system ends up, after a long excursion in phase space, in a stationary pattern with 12 modes and rectangular symmetry (last snapshot in Fig. 13). Note that two of the Fourier modes (the ones over the vertical axis) have the largest amplitude while the two perpendicular modes have a small intensity. All the other eight modes have the same intensity which is in between the values of the previous ones. This pattern, due to its rectangular symmetry in Fourier space, is not directly connected with the unstable manifold of the original hexagonal branch. It probably belongs to a separate branch emerging from the homogeneous solution. However, for some parameter range it seems to be an important attractor of the dynamics. As discussed below, this pattern is also often observed in the experiment. In larger systems, the

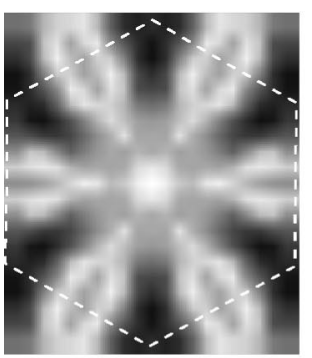

(a)

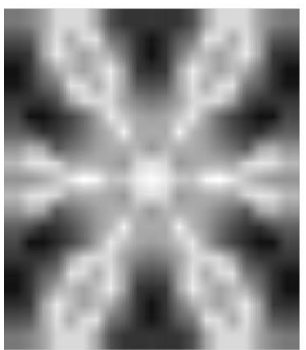

(b)

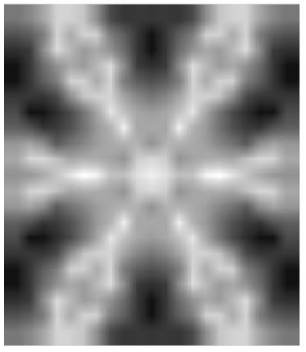

(c)
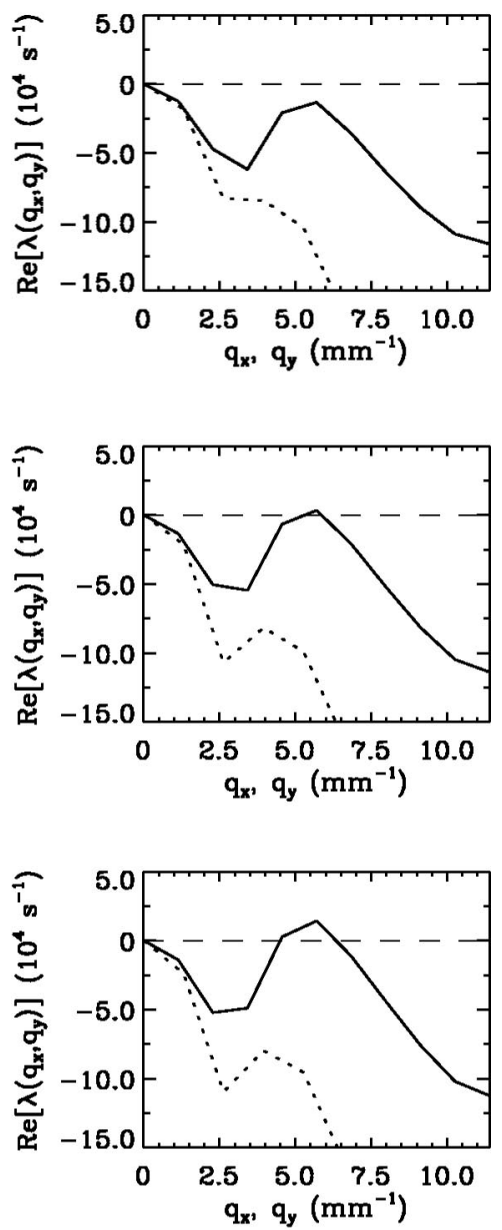

FIG. 10. The same as in Fig. 4 for a stationary hexagonal pattern with wave number $k=22.8 \mathrm{~mm}^{-1}$ crossing the $q=k / 4$ instability threshold located at $P_{k / 4}^{0}=2.75 \times 10^{5} \mathrm{~s}^{-1}$ (from top to bottom $P^{0}$ $=2.5 \times 10^{5} \mathrm{~s}^{-1}, 2.8 \times 10^{5} \mathrm{~s}^{-1}$, and $3.0 \times 10^{5} \mathrm{~s}^{-1}$ ).

nonlinear dynamics leads, in general, to a complex, nonstationary pattern with many excited Fourier components, which, however, resembles on average the one depicted in the last row of Fig. 13.

\section{F. Discussion of the theoretical results}

The analysis described above establishes that hexagonal patterns are stable only in a closed region of the $P^{0}-k$ diagram. It is the "hexagonal" analog of the "Busse balloon" for stripes [35,1]. At the sides, it is limited by longwavelength instabilities and the finite wavelength $q \sim k / \sqrt{3}$ and $q \sim k / 2$ instability, respectively. The long-wavelength instability results in the formation of a hexagonal pattern with a different stable wave number. The same is true for the $\sim k / \sqrt{3}$ instability for low pump values.

The upper boundary of the stability balloon is given by finite wavelength instabilities which result in the formation of more complex patterns consisting of 12 wave vectors (regions $q=k / 4, q=k / 2$ and $q \sim k / 2$ ). Only the $q=k / 2$ instability is supercritical. Hence the coexistence of hexagons and more complex patterns can be expected. 


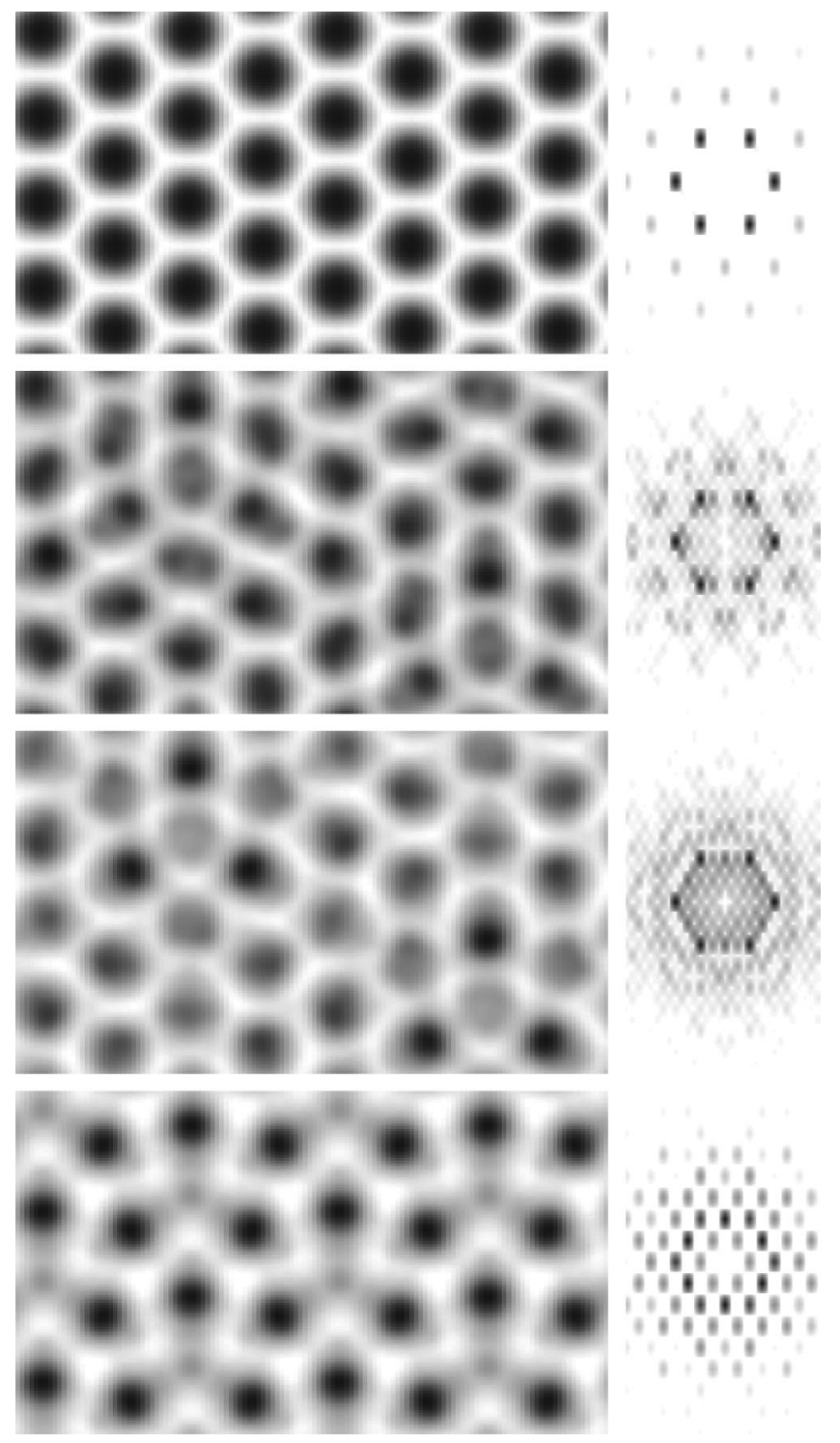

FIG. 11. The same as in Fig. 5 for a hexagonal pattern inside the $q=k / 4$ region $\left(k=22.8 \mathrm{~mm}^{-1}, P^{0}=3.0 \times 10^{5} \mathrm{~s}^{-1}\right)$. Note the final nonlinear selection of Fourier modes different from those that grow linearly. The final state is a pattern formed by 12 wave vectors.

In order to get some insight into the possibility of multistability between different patterns, additional simulations were performed on a square grid with periodic boundary conditions. Noise was added in order to shorten the transients. The simulations confirm that the hexagonal pattern formed spontaneously at threshold does not destabilize for a pump rate lower than $P^{0}=3.4 \times 10^{5} \mathrm{~s}^{-1}$, if the pump rate is increased. If the pump rate is decreased again, patterns with 12 Fourier components persist down to a level of about $P^{0}$ $\approx 1.7 \times 10^{5} \mathrm{~s}^{-1}$, i.e., $30 \%$ above the primary threshold. Starting from noisy initial conditions hexagons form up to a pump rate of typically $P^{0}=1.8 \times 10^{5} \mathrm{~s}^{-1}$ and patterns with 12 Fourier components form above this value. This transition level amounts to a pumping level of $38 \%$ above threshold. In most cases, if the size of the system is large enough, the resulting structures are somehow irregular and/or consist of

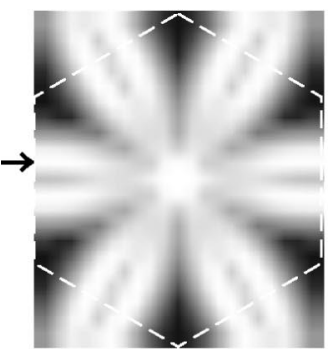

(a)

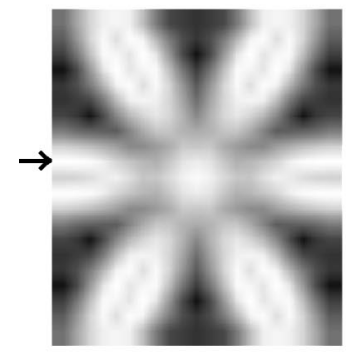

(b)

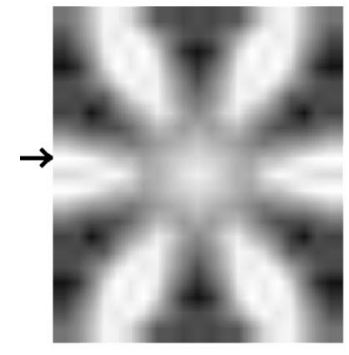

(c)
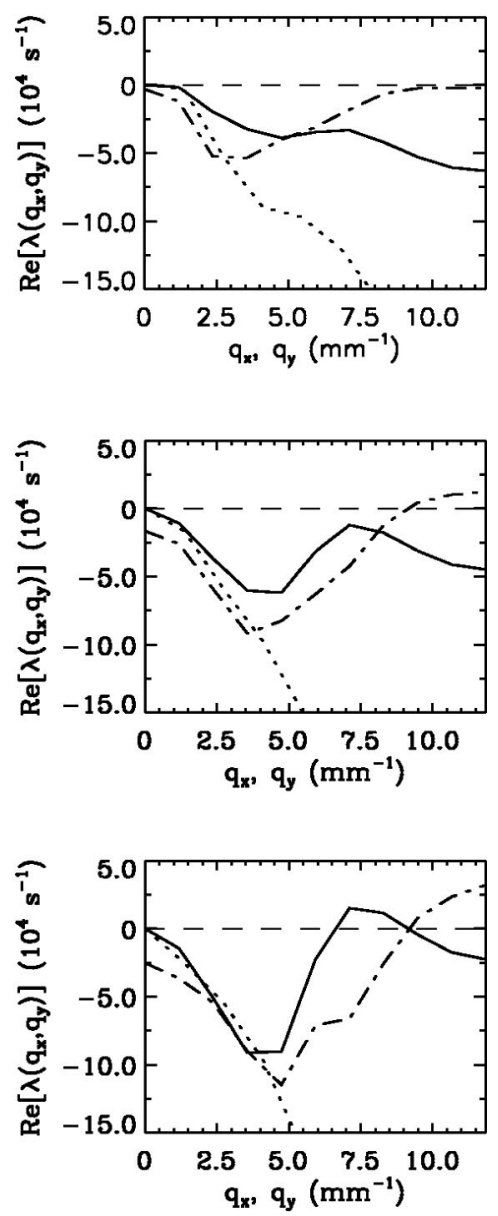

FIG. 12. The same as in Fig. 4 for a hexagonal pattern with wave number $k=23.7 \mathrm{~mm}^{-1}$ crossing the instability line $\left(P_{\sim k / 2}^{0}\right.$ $=1.65 \times 10^{5} \mathrm{~s}^{-1}$ ) of region $q \sim k / 2$. From top to bottom $P^{0}=1.6$ $\times 10^{5} \mathrm{~s}^{-1}, 2.0 \times 10^{5} \mathrm{~s}^{-1}$, and $2.5 \times 10^{5} \mathrm{~s}^{-1}$. The dot-dashed line corresponds to the transverse cut of $\operatorname{Re}\left[\lambda_{0}\left(q_{x}, q_{y}=1.37\right)\right]$ as indicated by the arrow on the left.

several domains of patterns with 12 wave vectors of different kind and/or orientation. In simulations performed for testing purposes (500 000 iterations or $50 \mathrm{~ms})$ a stationary state was not reached. However, the form of the patterns does not change qualitatively. At the present stage of the investigations it is not clear whether this is due to long lasting transients or whether there is an intrinsic time dependence. Typically, perfect stationary patterns are found, if a suitable seed is used. These results demonstrate that hexagons and more complex pattern coexist over a rather large range of pump values.

Finally, we comment on the relationship of the superlattice patterns obtained here to the ones observed in other pattern forming systems. Superlattices in a general sense are patterns with long-range periodicity. This implies that the Fourier components lie on a grid, whose meshes may be much finer than the length of the wave vectors. In the simplest case all $N$ fundamental wave vectors have equal lengths, but the rotational symmetry is $M$-fold only ( $M$ $<N)$. In the field of spontaneous pattern formation in dissipative systems this applied to the superlattices discussed by 


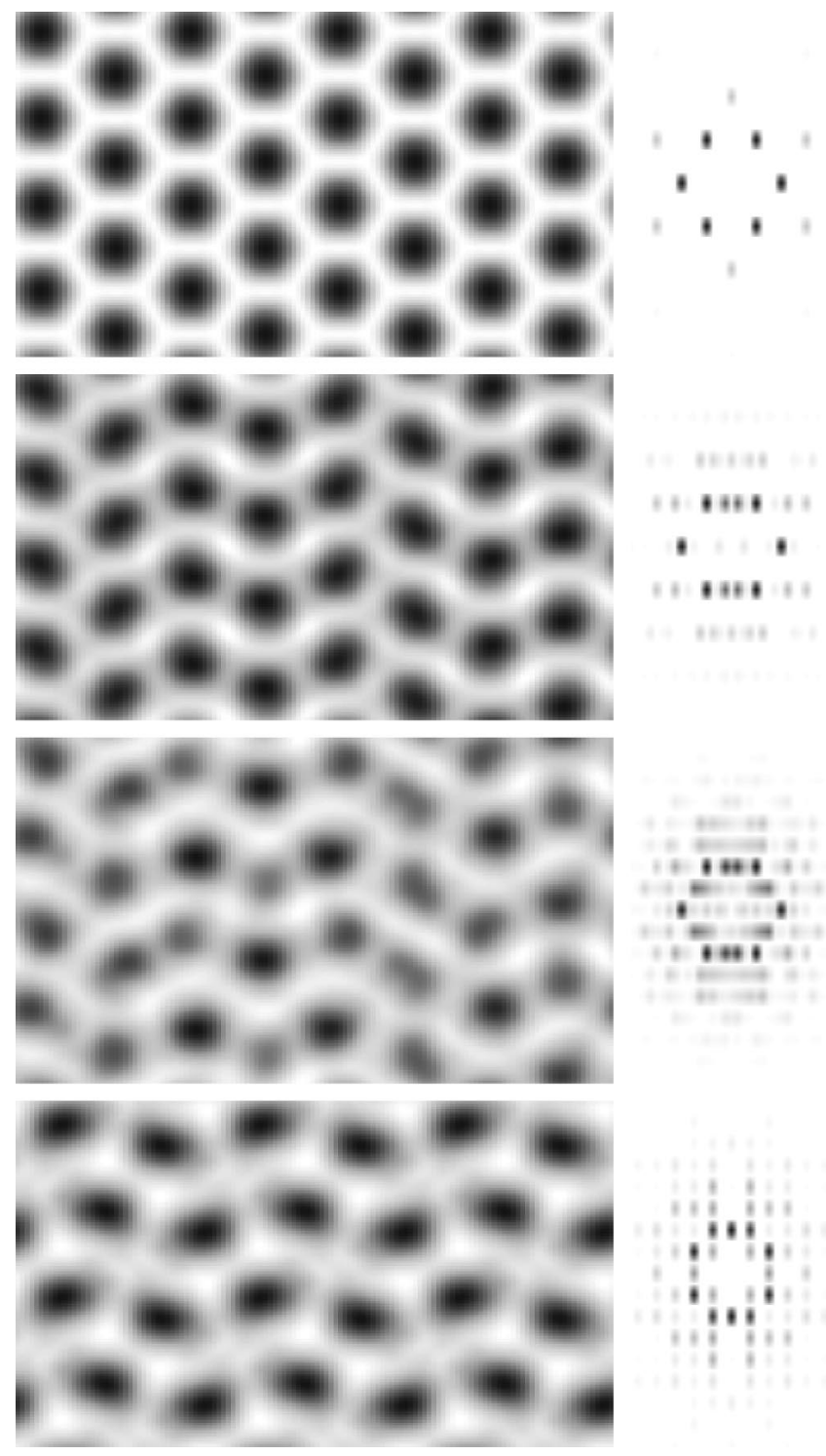

FIG. 13. The same as in Fig. 5 for a hexagonal pattern inside the $q \sim k / 2$ region $\left(k=23.7 \mathrm{~mm}^{-1}, P^{0}=2.5 \times 10^{5} \mathrm{~s}^{-1}\right)$. Note the linear growth of the wave vectors predicted by the stability analysis. The final stationary state with 12 modes and rectangular symmetry is reached after a long excursion in phase space.

Dionne et al. $[2,3]$. In the pioneering paper on the observation of superlattices in the Faraday instability by Kudrolli et al. [7] one of the patterns belongs to this type. It is built from two hexagonal triads having the same wave number and being rotated by an angle of about $22^{\circ}$ with respect to each other. It was termed "superlattice type I." In the same paper, the formation of a pattern termed "superlattice type II" was reported. This pattern consists of two hexagonal triads which are rotated by an angle of $30^{\circ}$ with respect to each other. The two triads have very different wave numbers (ratio $\sqrt{3}$ ). This ratio indicates that the inner set of wave vectors that defines the periodicity length might be interpreted as subharmonics of the driven fundamental wave vectors. Subsequently, several different realizations of superlattice patterns containing very different-often harmonic-wave
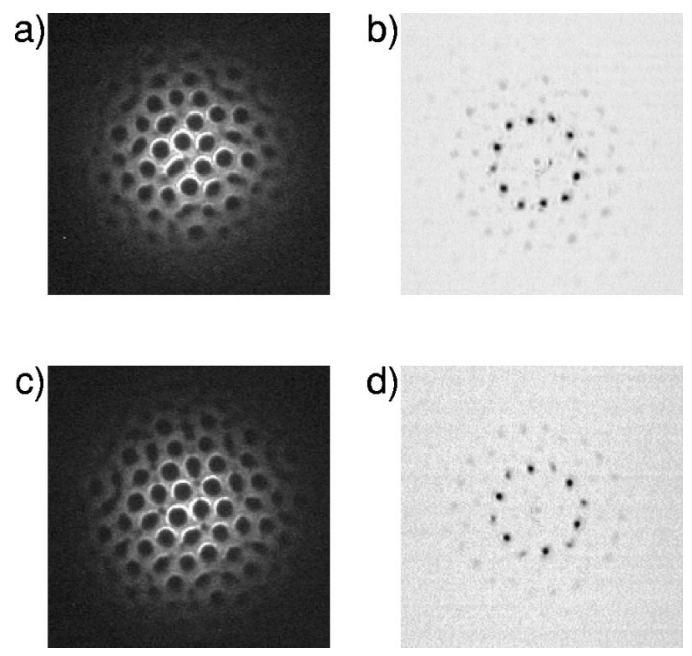

FIG. 14. Experimentally observed near (a,c) and far field (b,d) intensity distributions of the transmitted beam. Parameters: nitrogen buffer gas pressure $p_{\mathrm{N}_{2}}=200 \mathrm{hPa}$, sodium cell temperature $T$ $=318^{\circ} \mathrm{C}$, and distance between the sodium cell and the feedback mirror, $d=88 \mathrm{~mm}$; (a) input power $\mathcal{P}_{\text {in }}=113 \mathrm{~mW}$, detuning from the sodium $-D_{1}$ line $\Delta=3.6 \mathrm{GHz}$; (b) $\mathcal{P}_{\text {in }}=200 \mathrm{~mW}, \Delta=2.5 \mathrm{GHz}$.

numbers were observed by other groups in the Faraday instability $[8-10,36,37]$.

In the superlattice patterns discussed here, the wave numbers of the two constitutive triads differ, but only slightly, and they are certainly not harmonics of each other. Hence, these patterns differ from both superlattice patterns, type I and type II, and constitute a new type ("superlattice type III"). We conjecture that their formation is favored by the fact that the system is operated quite far from threshold so that the region of linear instability has a finite width.

It should be mentioned that patterns with a wave-vector configuration like that in Figs. 9 and 11, i.e., two hexagonal triads rotated by $30^{\circ}$ and having wave numbers different by $2 / \sqrt{3}$, are predicted to be among the ones emerging from "generic" bifurcations of hexagons [17]. In the latter work, the instabilities of hexagonal patterns are classified using Floquet theory and a group theoretical approach. We consider this coincidence as encouraging for future analytical studies of the mechanisms of these instabilities. The "quasisubharmonic" instabilities (i.e., the ones at $q \sim k / 2$ and $q$ $\sim k / \sqrt{3}$ ) are not captured by the analysis in Ref. [17], since the paper applies to a "strong resonance" and low-amplitude situation, i.e., the analysis is restricted to the minima of the Arnold tongues belonging to the subharmonic resonances [38].

\section{COMPARISON TO EXPERIMENT}

As discussed in Ref. [25], negative hexagons develop spontaneously from the unstructured state, if some threshold of input power is crossed. This is in agreement with the analysis of Sec. IV and previous numerical $[25,39]$ and analytical studies [39]. Above a secondary threshold, patterns consisting of 12 fundamental Fourier modes emerge $[26,13,14]$. Some examples are shown in Figs. 14 and 15. The transition occurs about $30-40 \%$ above threshold. This 

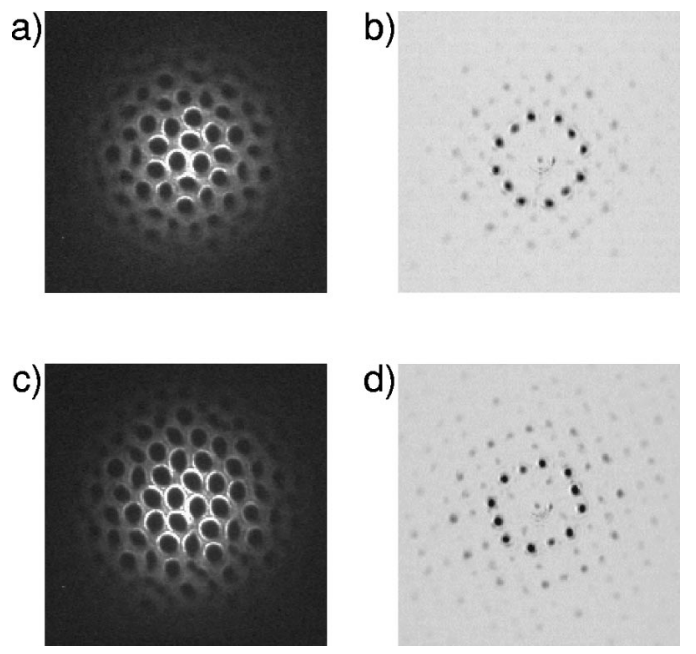

FIG. 15. Experimentally observed near (a,c) and far field (b,d) intensity distributions of the transmitted beam. Parameters as in Fig. 14(a), but in (b) $\mathcal{P}_{\text {in }}=188 \mathrm{~mW}$.

power level is considerably lower than the limit at which the hexagons should become linearly unstable (about 2.6 times threshold, Fig. 3), i.e., the secondary instabilities in the experiment take place at lower values of the pump power than in the theoretical analysis. This might have different reasons. First, the power level for the secondary instabilities in the experiment approximately matches the power level at which multistability between hexagons and secondary patterns appears in the simulations (see the preceding section). This observation gives some support to the hypothesis that noise-inevitably present in the experiment-might induce transitions to a coexistent state before the point of linear instability is reached. Second, due to the Gaussian profile of the input profile the boundary conditions differ in the experiment and in theory. Especially the fact that the bifurcation parameter has a spatial dependence might play a role in advancing (or delaying) secondary instabilities. These questions need further consideration in future work.

Patterns consisting of 12 Fourier modes are also obtained in the simulations as discussed above. In the following, we will compare the patterns observed in the experiment with the ones obtained in the simulations. However, one should keep in mind that the main aim of the theoretical investigations was to describe the onset of the instability of the hexagons and not a survey and classification of all possible asymptotic states. The preliminary simulations indicate a high level of multistability between different patterns, which make a determination of the complete solution space a formidable task which has to be postponed to forthcoming work.

In the experiment, the orientation is not directly accessible. The observed quantities are the intensity distribution of the transmitted field at the exit facet of the medium ("near field pattern") and the Fourier power spectrum of the transmitted field ("far field pattern"). The near field pattern can be calculated by

$$
I(x, y) \sim e^{-2 \alpha_{0} L[1-\phi(x, y)]},
$$

which has a monotonous but highly nonlinear dependence on $\phi(x, y)$. The far field pattern is obtained from

$$
I(\vec{k}) \sim\left|\int e^{-\alpha_{0} L(1-i \bar{\Delta})[1-\phi(\vec{x})]} e^{i \vec{k} \cdot \vec{x}} d \vec{x}\right|^{2} .
$$

In the parameter range considered before in the simulations and far enough above the secondary threshold, there is also in the experiment a region with multistability between different patterns consisting of twelve wave vectors (see Fig. 3 of Ref. [14]). Apart from quasipatterns with a 12-fold rotational symmetry (Figs. 3(a,b) of Ref. [14], see also Refs. $[26,20,13])$ and patterns in which neither in Fourier space nor in real space a particular order is apparent (Figs. 3(c,d) of Ref. [14]), there are two dominant types of patterns which have a Fourier power spectrum with a sixfold [Figs. 14(a,b)] or a fourfold rotational symmetry [Figs. 15(a,b)].

The latter pattern strongly resembles the result of the numerical simulation depicted in Fig. 13. It also consists of four rows with three wave vectors. The angle between adjacent rows is $90^{\circ}$. The experimentally observed and the numerically simulated patterns differ in the ratio of the wave numbers in the orthogonal directions. While the ratio is 1 within an accuracy of less than $2 \%$ in Figs. 15(a,b), the wave numbers differ by more than $10 \%$ in the numerical simulation (Fig. 13), i.e., the pattern is squeezed in comparison to the form observed experimentally. However, also in the experiment similar squeezed patterns occur for higher input power as depicted in Figs. 15(c,d). In this pattern the fourfold symmetry is clearly broken and the discussed ratio is 1.063. Therefore it resembles even more closely the pattern obtained numerically in Fig. 13. It is also apparent in the simulations as well as in the experiments that the wave vectors at the ends of the rows have approximately the same amplitude, whereas the pairs of wave vectors in the center of the rows have a lower or a higher amplitude than the former ones.

The numerically obtained pattern in Fig. 11 has the same wave-vector configuration as the experimentally observed pattern in Figs. 14(a,b), i.e., it consists of two hexagonal triads of slightly different wave numbers which are rotated by $30^{\circ}$. The triad with the larger wave number has a lower amplitude than the one with the smaller wave number. Nevertheless, differences in the near field patterns hint to the fact that the phases of the Fourier peaks are not the same. In order to clarify the deviation three generated patterns with the same Fourier spectrum but different phase distributions are shown in Fig. 16.

If all phases are $\pi$ as in Fig. 16(a) the near field obeys a sixfold rotational symmetry. The depicted structure resembles neither the experimental nor the numerical result. In the case of Fig. 16(b), in which the phases are $\pi / 3$ for all Fourier modes, the pattern has a threefold rotational symmetry. It is built by minima that constitute triangles. This structure is also obtained as the asymptotic pattern in Fig. 11.

The pattern observed in the experiment [Figs. 14(a,b)] resembles the structure depicted in Fig. 16(c). For this structure one phase in each triad is $\pi$, all others are zero. The resulting pattern has a twofold rotational symmetry in real 
a)

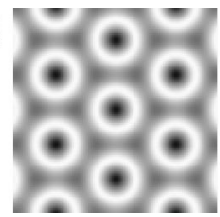

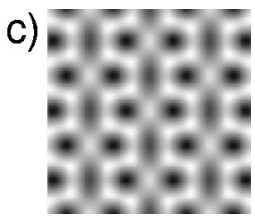

FIG. 16. Hexagonal superlattices generated by the addition of 12 Fourier modes with the same wave vectors and different phases: (a) all phases are $\pi$, (b) all phases are $\pi / 3$, and (c) one phase in each triad is $\pi$, all others are zero. The wave vectors are given by two hexagonal triads with different wave numbers that are mutually at an angle of $30^{\circ}$ (see, e.g., Fig. 11). The amplitude of the triad with the smaller wave number is twice the amplitude of the triad with the larger wave number.

space. The basic structure is a squeezed hexagon of which the center is built by an elongated minimum. This basic structure is typical for experimentally observed near field intensity distributions. However, for lower values of the detuning the phase distribution of the observed patterns is less clear. In this parameter region, some of the experimentally observed patterns [Figs. 14(c,d)] contain groups of triangular arrangements of three minima around a smaller central minima [Figs. 14(c,d)]. These patterns bear a quite close resemblance to the numerically obtained one depicted in Fig. 11 . We conclude that the phase selection within the hexagonal superstructure is weak.

\section{CONCLUSIONS}

We have analyzed in detail the secondary bifurcations of hexagonal patterns in a microscopic model for an alkali metal vapor in a single-mirror arrangement. The stability analysis of the stationary hexagonal patterns predicts different instabilities depending on their fundamental wave number. Hexagons with wave numbers much lower or much larger than the critical one undergo long-wavelength instabilities or instabilities at $q \sim k / \sqrt{3}$ or $q \sim k / 2$, while hexagonal patterns with a wave number close to the critical one are stable for moderate values of the pump and undergo finite wavelength instabilities (regions $q=k / 4, q=k / 2$, and $q$ $\sim k / 2$ ) for large values of the pump. After the instability has produced a growing disturbance, the crucial next step is some intrinsically nonlinear mechanism by which the system moves towards a new state. In some cases, the perturbation is found to saturate to finite amplitude and the new state resembles the unstable deformation of the original pattern. This is the case only for the $q=k / 2$ instability which result in the formation of a pattern with 12 wave vectors. In the other cases, the system undergoes a long excursion in the phase space until it reaches a new attractor. In some cases it is a hexagonal pattern with a different—stable-wave number or a periodic pattern (superlattices) with 12 wave vectors.

Since most of the important instabilities are subcritical, hexagons and superlattice patterns might coexist and there is also coexistence between different superlattice patterns. Due to the high level of multistability present it is very difficult to get an overview on all types of possible behavior and transition scenarios. This is especially true in a large system in which competition between several coexistent attractors might lead to long-lasting transients and nonstationary states.

Experimentally, secondary instabilities of the hexagons against more complex patterns consisting of 12 wave vectors were observed. There is a correspondence between some of the experimentally observed superlattice patterns and some of the patterns obtained numerically. In the experimental system noise induces transitions between the different coexisting multistable patterns. Also the Gaussian profile of the pump beam may affect the stability of some of the patterns. A thorough analysis of the asymptotic states is beyond the scope of the present paper and needs considerable further efforts.

The present analysis established the limits for the stability of hexagons and identified the modes relevant for the destabilization. Future work will be directed to a detailed investigation of the nonlinear evolution of the secondary instabilities and the asymptotic states, and needs to address the possible influence of further branches emerging from the homogeneous solutions.

\section{ACKNOWLEDGMENTS}

The authors acknowledge financial support from the European Science Foundation (PHASE network). D.G. and P.C. acknowledge financial support from MCyT (Spain, projects Acciones Integradas Grant Nos. HA98-29, PB97-0141-C0202, BFM2001-0341-C02-01, and BFM2000-1108). E.G.W., T.A. and W.L. acknowledge financial support by the Deutsche Akademische Austauschdienst (Acciones Integradas) and by the Deutsche Forschungsgemeinschaft. T.A. acknowledges fruitful discussions with L. Gil.
[1] M.C. Cross and P.C. Hohenberg, Rev. Mod. Phys. 65, 851 (1993).

[2] B. Dionne and M. Golubitsky, Z. Angew. Math. Phys. 43, 36 (1992).

[3] B. Dionne, M. Silber, and A.C. Skeldon, Nonlinearity 10, 321 (1997).

[4] M. Silber and M.R.E. Proctor, Phys. Rev. Lett. 81, 2450 (1998).

[5] D.P. Tse, A.M. Rucklidge, R.B. Hoyle, and M. Silber, Physica D 146, 367 (2000).
[6] S.L. Judd and M. Silber, Physica D 136, 45 (2000).

[7] A. Kudrolli, B. Pier, and J.P. Gollub, Physica D 123, 99 (1998).

[8] C. Wagner, H.W. Müller, and K. Knorr, Phys. Rev. Lett. 83, 308 (1999).

[9] C. Wagner, H.W. Müller, and K. Knorr, Phys. Rev. E 62, R33 (2000).

[10] H.-J. Pi, S.Y. Park, J. Lee, and K.J. Lee, Phys. Rev. Lett. 84, 5316 (2000).

[11] J.L. Rogers, M.F. Schatz, O. Brausch, and W. Pesch, Phys. 
Rev. Lett. 85, 4281 (2000).

[12] E. Pampaloni, S. Residori, S. Soria, and F.T. Arecchi, Phys. Rev. Lett. 78, 1042 (1997).

[13] T. Ackemann, E. Große Westhoff, M. Pesch, D. Rudolph, and W. Lange, Proc. SPIE 4751, 370 (2002).

[14] E. Große Westhoff, R. Herrero, T. Ackemann, and W. Lange, Phys. Rev. E 67, 025203 (2003).

[15] P. Coullet and G. Iooss, Phys. Rev. Lett. 64, 866 (1990).

[16] L. Gil, Physica D 147, 300 (2000).

[17] C. Pirat and L. Gil, Physica D 179, 92 (2003).

[18] G. D'Alessandro and W.J. Firth, Phys. Rev. Lett. 66, 2597 (1991).

[19] G. D’Alessandro and W.J. Firth, Phys. Rev. A 46, 537 (1992).

[20] T. Ackemann and W. Lange, Appl. Phys. B: Lasers Opt. 72, 21 (2001)

[21] A. Kastler, J. Opt. Soc. Am. 47, 460 (1957).

[22] F. Mitschke, R. Deserno, W. Lange, and J. Mlynek, Phys. Rev. A 33, 3219 (1986).

[23] T. Ackemann, A. Aumann, E. Große Westhoff, Yu.A. Logvin, and W. Lange, J. Opt. B: Quantum Semiclassical Opt. 3, S124 (2001).

[24] M. Le Berre, D. Leduc, E. Ressayre, A. Tallet, and A. Maitre, Opt. Commun. 118, 447 (1995).

[25] A. Aumann, E. Große Westhoff, R. Herrero, T. Ackemann, and
W. Lange, J. Opt. B: Quantum Semiclassical Opt. 1, 166 (1999).

[26] R. Herrero, E. Große Westhoff, A. Aumann, T. Ackemann, Yu.A. Logvin, and W. Lange, Phys. Rev. Lett. 82, 4627 (1999).

[27] The value of the asymptote has been numerically evaluated for the parameter values given in the caption of Fig. 2 .

[28] P. Borckmans, G. Dewel, A. D. Wit, and D. Walgraef, in Chemical Waves and Patterns, edited by R. Kapral and K. Showalter (Kluwer Academic, Dordrecht, 1995), pp. 323-363.

[29] D. Walgraef, in Spatio-Temporal Pattern Formation (SpringerVerlag, Berlin, 1997).

[30] D. Gomila and P. Colet, Phys. Rev. E 66, 046223 (2002).

[31] J. Lauzeral, D. Metens, and D. Walgraef, Europhys. Lett. 24, 707 (1993).

[32] M. Bestehorn, Phys. Rev. E 48, 3622 (1993).

[33] M.M. Sushchik and L.S. Tsimring, Physica D 74, 90 (1994).

[34] B. Echebarria and C. Pérez-García, Europhys. Lett. 43, 35 (1998).

[35] F.H. Busse, Rep. Prog. Phys. 41, 1929 (1978).

[36] H. Arbell and J. Fineberg, Phys. Rev. Lett. 81, 4384 (1998).

[37] J.L. Rogers, M.F. Schatz, J.L. Bougie, and J.B. Swift, Phys. Rev. Lett. 84, 87 (2000).

[38] L. Gil (Private communication).

[39] E. Große Westhoff, Ph.D. thesis, Westfälische WilhelmsUniversität Münster, 2002. 